\section{National consensus on Helicobacter pylori infection: the next-day challenge}

\author{
Apostolis Papaefthymiou ${ }^{a, b, c}$, \\ Michael Doulberis ${ }^{b, c, d}$, Stergios A. Polyzos ${ }^{b}$, \\ Jannis Kountouras
}

401 General Military Hospital of Athens, Greece; Aristotle University of Thessaloniki, Macedonia, Greece; Ippokration Hospital, Aristotle University of Thessaloniki, Macedonia, Greece; University of Zurich, Switzerland

The Hellenic Society of Gastroenterology [1] recently published the National consensus on Helicobacter pylori (H. pylori) infection, following the necessity of a uniform approach to yield optimal eradication rates. As implied by the recent Maastricht V/Florence consensus [2], an "add-on" strategy regarding antibiotics was adapted to overleap the increasing antibiotic resistance, combined with the absence of bismuth-containing drugs, thus perpetuating a "vicious circle" and emerging novel approaches in treatment models with a targeted pathophysiological perspective. Importantly, parameters connected with the multidrug resistance include the formation of $H$. pylori-related biofilms, suggesting the introduction of novel anti-biofilm therapeutic approaches using anti-biofilm agents [3].

A homophonous suggestion (Statement 10) proposed that $H$. pylori culture or molecular techniques should be conducted to evaluate the antimicrobial susceptibility. Nevertheless, those methods are characterized by limited availability in most regions. On the other hand, recent data imply a potential benefit of vitamin D (vitD) for H. pylori infection treatment, related to the vitD-receptor's (VDR) antimicrobial role [4-6]. VDR, stimulated by the active $H$. pylori infection, induces human $\beta$-defensins, which in high concentrations suppress $H$. pylori biofilm activity [3]; subtle or strong activation of VDR, due to vitD absence or adequacy, could contribute or not to $H$. pylori acclimatization, morbidity, resistance, and survival [3]. Additionally, vitD seems to act directly as an antibacterial agent through stimulation of defensins and cathelicidins, and vitD upregulated protein 1 (VDP1). possesses an H. pylori-specific antimicrobial ability, indicating a promising therapeutic potential $[7,8]$. Moreover, studies in mouse models revealed a protective role of a VDP1 against $H$. pylori-related gastric cancer [9]. Clinical studies concluded that vitD had a protective role against $H$. pylori infection and suggested its deficiency as a distinct risk factor in the failure of eradication treatment, while a recent metaanalysis concluded that vitD supplementation could change the effectiveness of eradication regimens [10]. Therefore, a National multicenter study has recently been inaugurated to elucidate the relationship between vitD and $H$. pylori infection and the potential beneficial effect of vitD supplementation during eradication treatment.

\section{References}

1. Georgopoulos SD, Michopoulos S, Rokkas T, et al. Hellenic consensus on Helicobacter pylori infection. Ann Gastroenterol 2020;33:105-124.

2. Malfertheiner P, Megraud F, O'Morain CA, et al; European Helicobacter and Microbiota Study Group and Consensus panel. Management of Helicobacter pylori infection-the Maastricht V/Florence Consensus Report. Gut 2017;66:6-30.

3. Kazakos EI, Dorrell N, Polyzos SA, Deretzi G, Kountouras J. Comment on "Effect of biofilm formation by clinical isolates of Helicobacter pylori on the efflux-mediated resistance to commonly used antibiotics". World J Gastroenterol 2017;23:6194-6196.

4. Wanibuchi $\mathrm{K}$, Hosoda $\mathrm{K}$, Ihara $\mathrm{M}$, et al. Indene compounds synthetically derived from vitamin $\mathrm{D}$ have selective antibacterial action on Helicobacter pylori. Lipids 2018;53:393-401.

5. Lu C, Yang J, Yu W, et al. Association between 25(OH)D level, ultraviolet exposure, geographical location, and inflammatory bowel disease activity: a systematic review and meta-analysis. PLoS One 2015;10:e132036.

6. Guo L, Chen W, Zhu H, et al. Helicobacter pylori induces increased expression of the vitamin $\mathrm{d}$ receptor in immune responses. Helicobacter 2014;19:37-47.

7. Wang TT, Nestel FP, Bourdeau V, et al. Cutting edge: 1,25-dihydroxyvitamin D3 is a direct inducer of antimicrobial peptide gene expression. J Immunol 2004;173:2909-2912.

8. Ramanathan B, Davis EG, Ross CR, Blecha F. Cathelicidins: microbicidal activity, mechanisms of action, and roles in innate immunity. Microbes Infect 2002;4:361-372.

9. Kwon HJ, Won YS, Nam KT, et al. Vitamin $\mathrm{D}_{3}$ upregulated protein 1 deficiency promotes $\mathrm{N}$-methyl-N-nitrosourea and Helicobacter pylori-induced gastric carcinogenesis in mice. Gut 2012;61:53-63.

10. Yang L, He X, Li L, Lu C. Effect of vitamin D on Helicobacter pylori infection and eradication: a meta-analysis. Helicobacter 2019;24:e12655.

aDepartment of Gastroenterology, 401 General Military Hospital

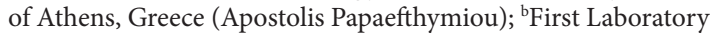
of Pharmacology, School of Medicine, Aristotle University of Thessaloniki, Macedonia, Greece (Apostolis Papaefthymiou, Michael Doulberis, Stergios A. Polyzos); ' Department of Internal Medicine, Second Medical Clinic, Ippokration Hospital, Aristotle University of Thessaloniki, Macedonia, Greece (Apostolis Papaefthymiou, Michael

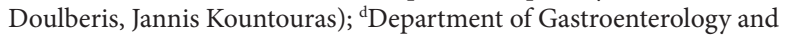
Hepatology, University of Zurich, Switzerland (Michael Doulberis)

\section{Conflict of Interest: None}

Correspondence to: Jannis Kountouras, Professor of Gastroenterology, Department of Internal Medicine, Second Medical Clinic, Ippokration Hospital, Aristotle University of Thessaloniki, Thessaloniki 54642, Macedonia, Greece, e-mail: jannis@auth.gr

Received 20 January 2020; accepted 5 February 2020; published online 27 March 2020

DOI: https://doi.org/10.20524/aog.2020.0469 